\title{
Crescimento e tolerância à salinidade em três espécies medicinais do gênero Plectranthus expostas a diferentes níveis de radiação
}

\author{
FREITAS, M.A.C.'; AMORIM, A.V.2; BEZERRA, A.M.E.'; PEREIRA, M.S.', BESSA, M.C.'; NOGUEIRA FILHO, \\ F.P.'; LACERDA, C.F.** \\ 'Universidade Federal do Ceará, Campus do Pici, bloco 804, CEP 60.455-760, Fortaleza-Brasil; ${ }^{\text {Universidade }}$ \\ da Integração Internacional da Lusofonia Afro-brasileira, Avenida da Abolição, número 3, Centro, 62790-000, \\ Redenção-Brasil; "cfeitosa@ufc.br
}

\begin{abstract}
RESUMO: No cultivo de plantas medicinais as condições de salinidade e de luz podem exercer influência no rendimento e na qualidade final da produção de biomassa. O objetivo deste trabalho foi avaliar o efeito de diferentes luminosidades e níveis de salinidade na água de irrigação sobre o crescimento e tolerância de três espécies de plantas do gênero Plectranthus. O ensaio foi conduzido em parcelas subsubdivididas com cinco repetições, sendo as parcelas referente ao fator ambiente (pleno sol e telado), as subparcelas aos níveis de salinidade na água de irrigação - CEa $\left(0,7 ; 1,9 ; 3,1 ; 4,3\right.$ e $\left.5,5 \mathrm{dS} \mathrm{m}^{-1}\right)$, e as subsubparcelas às três espécies do gênero Plectranthus ( $P$. amboinicus, $P$. barbatus e $P$. grandis). As variáveis de resposta foram: índice relativo de clorofila (IRC), área foliar (AF), razão de área foliar (RAF), área foliar específica (AFE), matéria seca da parte aérea (MSPA), matéria seca das raízes (MSR), matéria seca total (MST), relação MSR/MSPA, grau de tolerância à salinidade, e teor dos íons $\mathrm{Na}^{+} \mathrm{e} \mathrm{K}$. O estresse salino reduziu o crescimento das plantas, sendo as maiores reduções observadas nas plantas expostas a pleno sol. A salinidade influenciou a partição de matéria seca, sendo as raízes mais afetadas do que a parte aérea. Com o aumento da CEa houve aumento expressivo no teor foliar de $\mathrm{Na}^{+}$, enquanto o teor de $\mathrm{K}^{+}$e o IRC foram reduzidos. Entretanto, o acúmulo de $\mathrm{Na}^{+}$foi menor em $P$. grandis. Considerando-se a MST, verificou-se que as três espécies se mostraram moderadamente tolerantes à salinidade de até $3,1 \mathrm{dS} \mathrm{m}^{-1}$, exceto $P$. grandis cultivada em telado, classificada como tolerante. Em relação ao grau de redução na produção MSPA (parte de interesse comercial), poderia se recomendar o cultivo de $P$. grandis quando a água de irrigação contiver CEa de até $3,1 \mathrm{dS} \mathrm{m}^{-1}$.
\end{abstract}

Palavras-chave: estresse salino, íons inorgânicos, fatores ambientais, biomassa.

ABSTRACT: Growth and salinity tolerance in three medicinal species of the genus Plectranthus exposed to different levels of solar radiation. In the cultivation of medicinal plants, salinity and light can affect the yield and quality of biomass. The objective of this study was to evaluate the effect of light and salinity levels of the irrigation water on the growth and salt tolerance of three medicinal plant species of the genus Plectranthus. A completely randomized split-plot design with five repetitions was used. The plots were formed by the environment of cultivation (full sunlight and greenhouse), the subplots by the salinity in the irrigation water - ECw $\left(0.7,1.9,3.1,4.3\right.$ and $\left.5.5 \mathrm{dS} \mathrm{m}^{-1}\right)$, and the subsubplots by the three plant species $(P$. amboinicus, $P$. barbatus and $P$. grandis). The following variables were evaluated: relative chlorophyll index $(R C I)$, leaf area (LA), leaf area ratio (LAR), specific leaf area (SLA), shoot dry mass (SDM), root dry mass (RDM), total dry mass (TDM), RDM/SDM ratio, relative salt tolerance, and concentration of $\mathrm{Na}+$ and $\mathrm{K}+$ in leaves. Salinity reduced plant growth, with the greatest reductions observed in plants exposed to full sunlight. Salinity affected the dry matter partitioning, and the roots were more affected than the shoot. With increasing $\mathrm{ECw}$, there was a significant increase in the concentration of $\mathrm{Na}+$ in the leaves, while $\mathrm{K}+$ and $\mathrm{RCl}$ decreased. However, the increase in $\mathrm{Na}+$ accumulation was lower in $P$ grandis. The three species were moderately tolerant to salinity up to $3.1 \mathrm{dS} \mathrm{m}^{-1}$, considering the total dry mass production, except the $P$. grandis grown in greenhouse, classified as tolerant. Regarding the relative reduction in shoot dry mass (part of commercial interest), we could recommend the cultivation of $P$. grandis when the available irrigation water presents values of ECw up to $3.1 \mathrm{dS} \mathrm{m}^{-1}$.

Keywords: salt stress, inorganic ions, environmental factors, dry biomass. 


\section{INTRODUÇÃO}

A salinidade é um problema mundial que resulta na redução do potencial produtivo da maioria das culturas. Este problema é mais grave em regiões áridas e semiáridas, incluindo áreas no Nordeste brasileiro, onde a salinidade é um dos principais fatores limitantes para a produtividade agrícola (Silveira et al., 2012). O desempenho das plantas em condições salinas é influenciado pelo efeito osmótico e iônico, os quais promovem modificações nas atividades metabólicas das células e no processo de alongamento celular, afetando o crescimento da planta e, em casos extremos, pode ocasionar a morte do organismo como um todo (Saíram \& Tyagi, 2004; Taiz \& Zeiger, 2013).

Entretanto, a resposta das plantas à salinidade é dependente da espécie, do genótipo, do estádio fenológico de um mesmo genótipo e do período de exposição ao sal. As diferenças entre espécies estão associadas ao desenvolvimento de mecanismos fisiológicos e bioquímicos, como o ajustamento osmótico, alterações nas vias fotossintéticas, síntese de osmólitos compatíveis e ativação de sistemas antioxidantes enzimáticos e não enzimáticos, que aumentam a capacidade de sobrevivência das plantas em ambientes com concentrações elevadas de sais, os quais facilitam a absorção e retenção de água, protegem as funções dos cloroplastos e mantêm a homeostase iônica (Parida \& Das, 2005; Munns \& Tester, 2008).

O estresse salino é acentuado pela presença de fatores como déficit hídrico, altas temperaturas e luminosidade, expondo, assim, as plantas, a uma pressão adaptativa adicional (Debez et al., 2008; Silveira et al., 2012). Particularmente em ambientes áridos e semiáridos, o estresse salino associado à alta luminosidade pode comprometer a produtividade de culturas não adaptadas, constituindo-se em uma combinação de fatores estressantes, notadamente para as espécies C3. Portanto, a obtenção do conhecimento acerca das melhores condições de cultivo e identificação do grau de tolerância de espécies à salinidade é necessária para o aproveitamento de águas e solos afetados por sais e para expansão agrícola nessas regiões.

Embora sejam conhecidas informações sobre o crescimento e a fisiologia de plantas de interesse econômico, cultivadas em ambientes nos quais são submetidas ao estresse salino e a restrição de luminosidade, pouco se sabe sobre o efeito dessas condições ambientais, isoladas ou combinadas, no rendimento de plantas medicinais, em especial do gênero Plectranthus, que contempla importantes espécies de uso medicinal no Nordeste brasileiro.

O gênero Plectranthus (Família Lamiaceae), encontrado na África, América, Oceania e Ásia, é constituído por espécies ornamentais, comestíveis e medicinais, sendo considerado um dos gêneros mais ricos em óleos essenciais, tendo como principais constituintes os monos e sesquiterpenos. O uso medicinal das espécies do gênero Plectranthus responde por aproximadamente $85 \%$ de todos os seus usos, dos quais $65 \%$ são representados pelas espécies $P$. amboinicus e $P$. barbatus (Lukhoba et al., 2006).

Os efeitos farmacológicos de algumas espécies do gênero Plectranthus têm sido demonstrados em trabalhos apresentados por Abdel-Mogib et al. (2002), Alasbahi \& Melzig (2010), Bandeira et al. (2011) e Rice et al. (2011), explicando a importância e larga utilização na medicina popular. No Brasil, as espécies desse gênero, conhecidas como boldo, são utilizadas e estudadas em relação as suas propriedades antidispépticas, analgésicas e estimulantes da digestão (Bandeira et al., 2011). Porém os efeitos de estresses ambientais, tais como o estresse salino e o excesso de radiação, no rendimento e na qualidade da matéria prima dessas plantas são pouco conhecidos.

Assim, avaliou-se o crescimento e a tolerância à salinidade em três espécies medicinais do gênero Plectranthus submetidas a níveis crescentes de condutividade elétrica na água de irrigação e a diferentes níveis de luminosidade.

\section{MATERIAL E MÉTODO}

O experimento foi realizado entre os meses de agosto e dezembro de 2011 no Núcleo de Ensino e Pesquisa em Agricultura Urbana (NEPAU) da Universidade Federal do Ceará - UFC, situada em Fortaleza - CE, localizada na latitude $3 \circ 44^{\prime} \mathrm{S}$, longitude $38^{\circ} 33^{\prime} \mathrm{W}$, na altitude de $20 \mathrm{~m}$, com três espécies medicinais do gênero Plectranthus Plectranthus amboinicus (Lour.) Spreng (malvarisco), Plectranthus barbatus (Andr.) Spreng (malva santa, boldo brasileiro) e Plectranthus grandis (Kramer) (boldo gigante).

O delineamento experimental foi inteiramente casualizado com parcelas subsubdivididas e cinco repetições (duas plantas por repetição), sendo as parcelas referentes ao fator ambiente (pleno sol e telado com redução de $50 \%$ da radiação incidente), as subparcelas referentes aos níveis de salinidade na água de irrigação - CEa $(0,7 ; 1,9 ; 3,1 ; 4,3$ e 5,5 dS $\left.\mathrm{m}^{-1}\right)$, e as subsubparcelas relacionadas às três espécies do gênero Plectranthus ( $P$. amboinicus, $P$. barbatus e $P$. grandis).

As plantas foram selecionadas de mudas produzidas através de propagação vegetativa, por

Rev. Bras. PI. Med., Campinas, v.16, n.4, p.839-849, 2014. 
estaquia, com matrizes pertencentes ao NEPAU e ao Horto de Plantas Medicinais da UFC. A propagação das mudas foi realizada entre os dias 30 de agosto e 1 de setembro de 2011. Para o enraizamento, as estacas foram colocadas em sacos de polietileno contendo, como substrato, uma mistura de arisco + húmus de minhoca, na proporção de 2:1, onde permaneceram em casa de vegetação por período de 40 dias para a formação das mudas. As características químicas do substrato foram: $\mathrm{pH}$ em $\mathrm{H}_{2} \mathrm{O}(1: 2,5)=6,7 ; \mathrm{Ca}^{2+}=5,1 \mathrm{cmol}_{c} / \mathrm{dm}^{3} ; \mathrm{Mg}^{2+}=$ $3,6 \mathrm{cmol}_{\mathrm{c}} / \mathrm{dm}^{3} ; \mathrm{Na}^{+}=1,39 \mathrm{cmol}_{\mathrm{c}} / \mathrm{dm}^{3} ; \mathrm{K}^{+}=2,15 \mathrm{cmol}_{\mathrm{c}} /$ $\mathrm{dm}^{3} ; \mathrm{SB}=12,25 \mathrm{cmol}_{\mathrm{c}} / \mathrm{dm}^{3} ; \mathrm{H}+\mathrm{Al}=1,65 \mathrm{cmol}_{\mathrm{c}} / \mathrm{dm}^{3}$; $\mathrm{Al}^{3+}=0,00 ; \mathrm{V} \%=88,13 ; \mathrm{P}=493,84 \mathrm{mg} / \mathrm{dm}^{3} ; \mathrm{C}=$ $1,43 \%$; M.O. $=2,46 \%$. As análises foram realizadas conforme Embrapa (1997).

Mudas enraizadas com 40 dias de idade foram transplantadas para vasos plásticos com capacidade para 7,5 litros, contendo o mesmo substrato utilizado para a formação da muda. Após o transplantio as mudas permaneceram durante 10 dias em telado com $50 \%$ de luminosidade, a fim de se restabelecerem do estresse sofrido no transplantio e passassem a emitir novas raízes e folhas. No dia 14 de outubro de 2011, as plantas foram expostas a dois níveis de radiação $(50 \%$ de luminosidade e pleno sol), onde foram submetidas aos tratamentos com água salina por um período de 60 dias. Os valores médios de temperatura e umidade relativa do ar foram de $27,32^{\circ} \mathrm{C}$ e $73,5 \%$, respectivamente.

Para o preparo das soluções foram utilizados os sais de $\mathrm{NaCl}, \mathrm{CaCl}_{2} \cdot 2 \mathrm{H}_{2} \mathrm{O}$ e $\mathrm{MgCl}_{2} \cdot 6 \mathrm{H}_{2} \mathrm{O}$, na proporção equivalente a 7:2:1, entre os cátions $\mathrm{Na}^{+}: \mathrm{Ca}^{2+}: \mathrm{Mg}^{2+}$, obedecendo-se a relação entre CEa e sua concentração $\left(\mathrm{mmol}_{\mathrm{c}} \mathrm{L}^{-1}=\mathrm{CE} \times 10\right)$, conforme Rhoades et al. (2000). Aágua foi aplicada de forma localizada, a cada dois dias, mantendo-se o solo na capacidade de campo e adicionando-se uma fração de lixiviação de 0,15 para que a água percolasse pelo fundo dos vasos de acordo com Ayers \& Westcot (1999), a fim de evitar o acúmulo excessivo de sais.

As variáveis avaliadas foram: índice relativo de clorofila (IRC) estimado em medidor portátil SPAD 502 (Minolta); área foliar (AF), determinada em medidor de superfície (LI - 3100, Área Meter, LiCor, Inc., Lincoln, Nebraska, USA); matéria seca da parte aérea (MSPA), composta de folhas e hastes, e matéria seca das raízes (MSR). A matéria seca (expressa em g planta-1) foi obtida após secagem em estufa, com circulação forçada de ar, a $75^{\circ} \mathrm{C}$, até massa constante. Com os dados de matéria seca e área foliar determinaram-se a razão de área foliar (RAF) pela relação área foliar/matéria seca total e a área foliar específica (AFE) pela relação área foliar/ matéria seca das folhas (Benincasa, 2003).
Para a determinação dos íons $\mathrm{Na} \cdot \mathrm{e} \mathrm{K}^{+}$, as amostras das folhas secas em estufa foram trituradas em moinho tipo Willey e armazenadas em sacos plásticos à temperatura ambiente. A extração do material vegetal foi realizada conforme a metodologia de Cataldo et al. (1975). Os íons $\mathrm{Na}^{+}$ e $\mathrm{K}^{+}$foram determinados em fotômetro de chama (Malavolta et al., 1989), calibrado com solução padrão de 20 ppm de $\mathrm{Na}^{+}$e 20 ppm de $\mathrm{K}^{+}$.

Os dados foram submetidos à análise de variância estudando-se a interação entre fatores. Os dados relativos à salinidade na água de irrigação e suas interações significativas foram desdobrados em análise de regressão sendo o modelo escolhido pelo $R^{2}$ ajustado. As análises estatísticas foram realizadas com o auxílio do software Sistema de Análise de Variância para Dados Balanceados (SISVAR), desenvolvido por Ferreira (2000).

Com as curvas de regressão obtidas para os dados de matéria seca foram calculadas as perdas percentuais em relação ao menor nível de salinidade (redução em \%). As perdas relativas foram utilizadas como índices para comparar a tolerância das três espécies estudadas, conforme Fageria et al. (2010), sendo a classificação feita separadamente para cada ambiente.

\section{RESULTADO E DISCUSSÃO}

A análise de variância para as variáveis de crescimento e partição de fotoassimilados na planta encontra-se representada na Tabela 1. Com exceção da MSPA e da MST que não foram influenciadas pelo fator ambiente, verificou-se efeito significativo dos fatores isolados (ambiente, salinidade e espécie) e da interação tripla sobre as demais variáveis estudadas. As interações duplas não apresentaram efeito significativo sobre as variáveis MSPA e MSR/ MSPA. A MST, também, não foi influenciada pela interação ambiente vs salinidade (Tabela 1).

Na Figura 1 observa-se a área foliar, razão de área foliar e área foliar específica das espécies em função da CEa e de diferentes níveis de luminosidade. A redução da área foliar em função do aumento da CEa, observada na Figura $1 \mathrm{~A}$ e $\mathrm{B}$, constitui uma das respostas iniciais da planta ao estresse salino e tem sido atribuída à diminuição na divisão celular e expansão da superfície da folha. Tal resposta reduz a capacidade de produção de fotoassimilados, limitando a produtividade da planta (Parida \& Das, 2005; Taiz \& Zeiger, 2010). Resultados semelhantes para plantas medicinais foram apresentados por Koyro (2006) em Plantago coronopus, Bernstein et al. (2010) em Ocimum basilicum, por Sabra et al. (2012), em espécies de Echinacea, e por Tounekti et al. (2012) em Salvia officinalis. 
TABELA 1. Resumo da análise de variância para área foliar (AF), razão de área foliar (RAF), área foliar específica (AFE), matéria seca da parte aérea (MSPA), matéria seca das raízes (MSR), matéria seca total (MST) e relação MSR/MSPA em três espécies medicinais do gênero Plectranthus cultivadas em telado com $50 \%$ de luminosidade e pleno sol, e submetidas a níveis crescentes de condutividade elétrica na água de irrigação. (Fortaleza-CE, UFC, 2012).

\begin{tabular}{|c|c|c|c|c|c|c|c|c|}
\hline \multirow{2}{*}{ FV } & \multirow{2}{*}{$\mathrm{GL}$} & \multicolumn{7}{|c|}{ Quadrados médios } \\
\hline & & $\mathrm{AF}$ & RAF & AFE & MSPA & MSR & MST & MSR/MSPA \\
\hline A & 1 & 4658655,91“ & $1,57^{\prime \prime}$ & $12,08^{\prime \prime}$ & $3,10^{\mathrm{NS}}$ & $7,51^{\circ}$ & $20,29^{\mathrm{Ns}}$ & $0,01^{* *}$ \\
\hline $\operatorname{Res}_{(a)}$ & 8 & 411296,5 & 0,07 & 0,66 & 7,3 & 0,71 & 10,35 & 0,001 \\
\hline B & 4 & $30506797,15^{*}$ & $0,10^{\text {NS }}$ & 2,56 & $873,62 *$ & 200,85 & $1882,95 *$ & 0,18 \\
\hline$A \times B$ & 4 & $2353063,78^{* *}$ & $0,66^{* *}$ & $5,66 \cdots$ & $6,88^{\mathrm{Ns}}$ & $5,14^{* *}$ & $21,88^{*}$ & 0,007 Ns \\
\hline $\operatorname{Res}_{(b)}$ & 32 & 535718,5 & 0,07 & 0,76 & 4,61 & 1,1 & 6,67 & 0,003 \\
\hline C & 2 & $62847395,88^{*}$ & $5,79 \cdots$ & $18,72^{*}$ & 470,02 & 102,72 & $1010,97^{* *}$ & $0,08^{*}$ \\
\hline$A \times C$ & 2 & $7020128,90 *$ & 1,56 & $13,3^{\cdots}$ & $14,05^{\circ}$ & $4,799^{*}$ & $9,62^{\text {Ns }}$ & 0,01 Ns \\
\hline$B \times C$ & 8 & $2884369,98 *$ & $0,41^{\cdots}$ & $1,76^{*}$ & $16,12^{*}$ & $4,23 \cdots$ & $33,60^{* *}$ & 0,004 Ns \\
\hline$A \times B \times C$ & 8 & $2362204,67^{*}$ & 0,40 " & $2,56 \cdots$ & $9,93^{\circ}$ & $7,34 *$ & $18,67^{* *}$ & $0,02^{* *}$ \\
\hline $\operatorname{Res}_{(c)}$ & 80 & 410672 & 0,06 & 0,52 & 3,69 & 0,97 & 4,02 & 0,004 \\
\hline $\mathrm{CV}_{(\mathrm{a})}(\%)$ & - & 25,3 & 24,1 & 31,21 & 16,34 & 19,95 & 15,31 & 16,24 \\
\hline $\mathrm{CV}_{(\mathrm{b})}(\%)$ & - & 28,88 & 23,98 & 33,61 & 12,98 & 23,5 & 12,29 & 24,94 \\
\hline $\mathrm{CV}_{(\mathrm{c})}(\%)$ & - & 25,28 & 21,03 & 27,73 & 11,62 & 22,09 & 9,55 & 28,18 \\
\hline
\end{tabular}

FV - fontes de variação; GL - graus de liberdade; A - ambiente; B - salinidade; C - espécies; CV - coeficiente de variação; ", ', Ns significativo pelo teste $\mathrm{F}$ a $1 \%, 5 \%$ e não significativo, respectivamente.

$P$. amboinicus e P. grandis tiveram sua área foliar ajustada a um modelo quadrático, observando, para a primeira espécie, a redução da área foliar a partir da CEa de $0,7 \mathrm{dS} \mathrm{m}^{-1}$, enquanto $P$. grandis apresentou aumento da área foliar até a CEa de 1,8 dS $\mathrm{m}^{-1}$, com redução drástica para os níveis de CEa seguintes (Figura 1A). Em pleno sol, a área foliar das três espécies reduziu linearmente com o aumento da CEa, observando que o grau de redução foi maior nas espécies $P$. amboinicus e $P$. grandis, que reduziram a área foliar a taxas constantes de 797,92 e $768,75 \mathrm{~cm}^{2}$ por unidade de CEa, respectivamente (Figura 1B).

A CEa não influenciou a razão de área foliar de $P$. grandis e $P$. barbatus cultivadas em telado (Figura $1 \mathrm{C}$ ), indicando que o efeito do estresse salino na área foliar dessas espécies foi da mesma intensidade que na matéria seca. Já na espécie $P$. amboinicus, cultivada em telado, se observou aumento da razão de área foliar a partir da CEa de 3,1 dS $\mathrm{m}^{-1}$ (Figura 1C). Em pleno sol houve redução linear da razão de área foliar $P$. grandis e $P$. barbatus em função do acréscimo da CEa, não sendo observada alteração na espécie $P$. amboinicus (Figura 1D). O decréscimo na razão de área foliar de $P$. barbatus e $P$. grandis cultivadas em pleno sol pode indicar que estas espécies apresentaram maior eficiência fotossintética, visto que nessas condições houve maior aproveitamento da área foliar fotossintetizante para a produção de matéria seca.

A área foliar específica de $P$. barbatus cultivada em telado aumentou linearmente em função da CEa, indicando que neste ambiente houve aumento da expansão foliar e redução na matéria seca das folhas. Resposta similar foi observada para $P$. amboinicus a partir da CEa de $3,1 \mathrm{dS} \mathrm{m}^{-1}$, não sendo observadas alterações na espécie $P$ grandis nas mesmas condições de cultivo (Figura 1E). Por outro lado, o aumento da salinidade no ambiente a pleno sol provocou redução da área foliar específica nas plantas de $P$. amboinicus e $P$. grandis, e não alterou a área foliar específica na espécie $P$. barbatus (Figura 1F).

O uso da água com níveis crescentes de condutividade elétrica promoveu reduções significativas na matéria seca das plantas, sendo o efeito negativo do sal mais pronunciado na MSR quando comparado à MSPA (Figura 2). A MSPA de $P$. amboinicus e $P$. barbatus reduziu linearmente com o aumento da CEa, com perdas de 3,03 e 1,89 g planta-1 por unidade de CEa, em telado, e de 2,72 e 3,01 g planta-1 $^{-1}$ por unidade de CEa em pleno sol (Figura 2A e B). Já, $P$. grandis apresentou ajuste quadrático, com maior acúmulo de MSPA quando submetida à CEa de 1,3 dS m-1 (Figura 2A e B). 

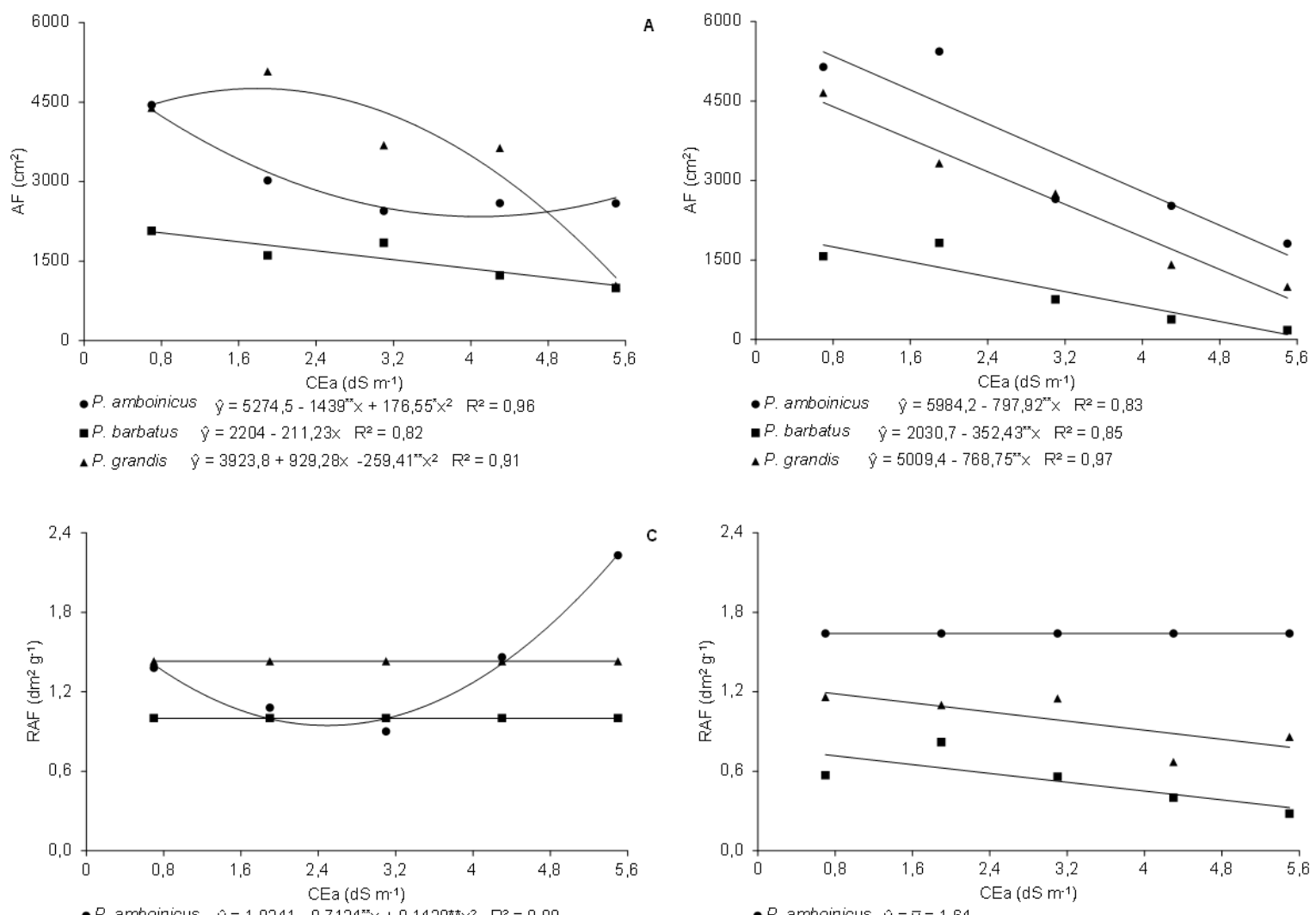

-P. amboinicus $\hat{y}=1,8341-0,7124^{* \pi} x+0,1429^{* \star} x^{2} \quad R^{2}=0,98$

-P amboinicus $\hat{\gamma}=\bar{\gamma}=1,64$

- $P$. barbatus $\hat{y}=0,7843-0,0833^{x} \times R^{2}=0,60$
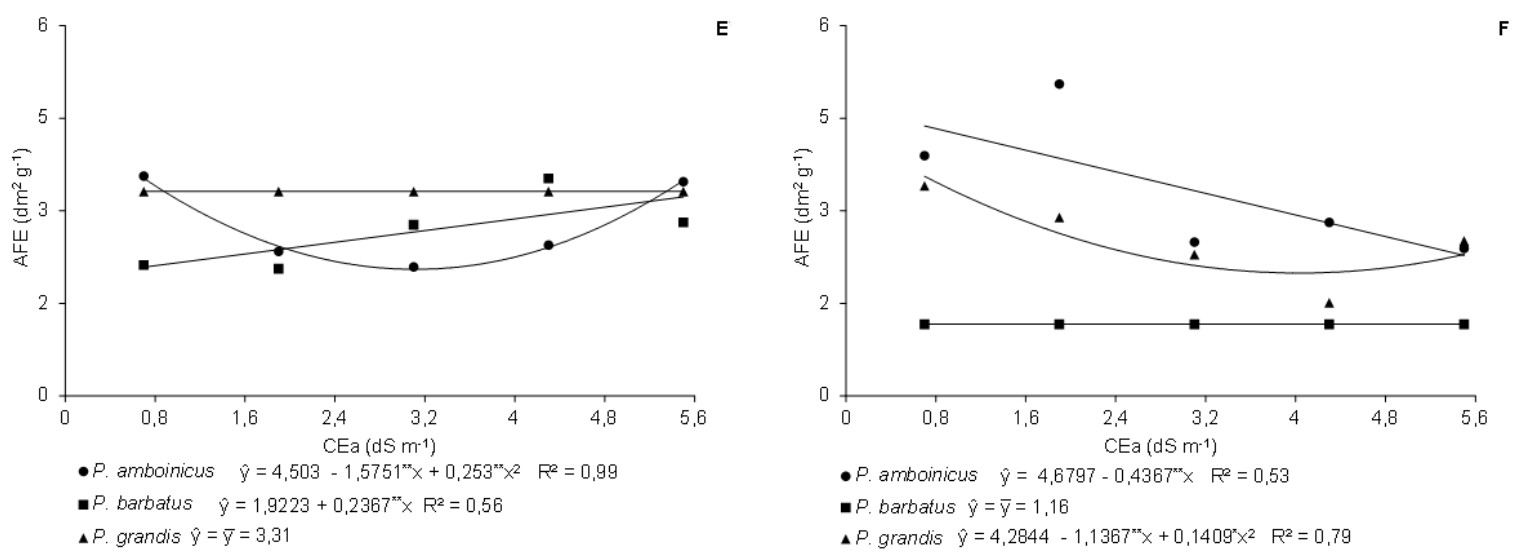

FIGURA 1. Área foliar (AF) (A) e (B), razão de área foliar (RAF) (B) e (C) e área foliar específica (AFE) (E) e $(F)$ em três espécies medicinais do gênero Plectranthus cultivadas em telado com $50 \%$ de luminosidade $(A)$, (C) e (E) e em pleno sol (B), (D) e (F) e submetidas a níveis crescentes de condutividade elétrica na água de irrigação (Fortaleza-CE, UFC, 2012).

A MSR de $P$. barbatus e $P$. amboinicus reduziu linearmente com o aumento da $\mathrm{CEa}$, tanto em telado, quanto a pleno sol (Figura $2 \mathrm{C} \mathrm{e} \mathrm{D).} \mathrm{No}$ entanto, estas espécies obtiveram redução máxima na MSR com o aumento da CEa de 0,7 para 5,5 dS $\mathrm{m}^{-1}$ a pleno sol, com perda na ordem de $99,43 \%$ para P. barbatus e $91,76 \%$ para P. amboinicus (Figura 2D). Contrário à condição ambiental de pleno sol, em telado a MSR de $P$. grandis apresentou maior redução em relação ao controle apenas nas CEa 4,3 e $5,5 \mathrm{dS} \mathrm{m}^{-1}$, com valores na ordem de $46,13 \%$ e $88,13 \%$, respectivamente (Figura $2 \mathrm{C} \mathrm{e} \mathrm{D)}$ ), indicando que a restrição parcial de luz pode ter induzido a melhor aclimatação dessas plantas nos níveis mais baixos de CEa.

A inibição do crescimento das plantas em 

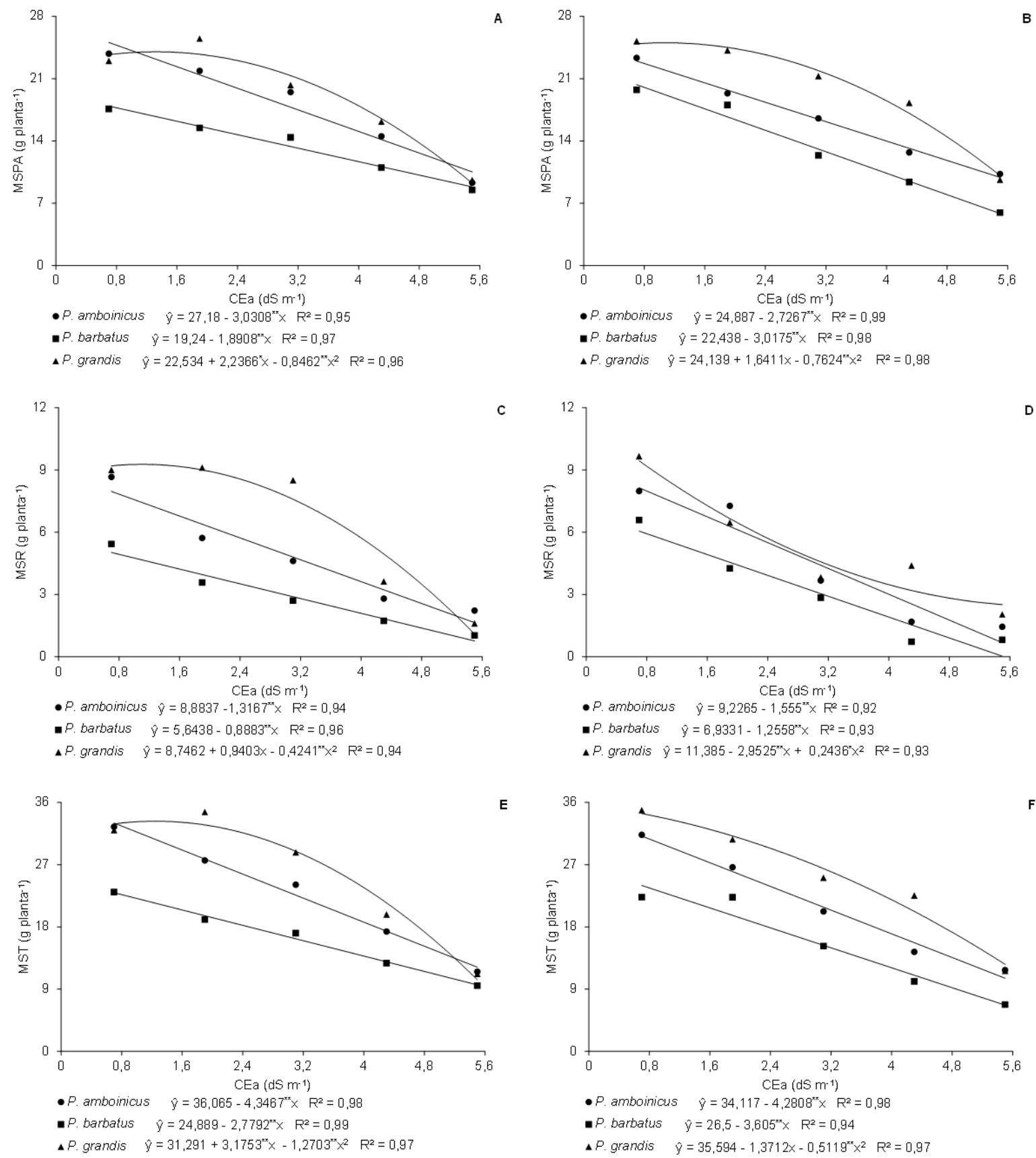

FIGURA 2. Matéria seca da parte aérea (MSPA) (A) e (B), matéria seca radicular (MSR) (B) e (C) e relação matéria seca raiz/matéria seca parte aérea (MSR/MSPA) (E) e (F) em três espécies medicinais do gênero Plectranthus cultivadas em telado com $50 \%$ de luminosidade (A), (C) e (E) e em pleno sol (B), (D) e (F) e submetidas a níveis crescentes de condutividade elétrica na água de irrigação (Fortaleza-CE, UFC, 2012).

ambientes com salinidade pode ser atribuída ao déficit hídrico causado pelo excesso de sais solúveis na zona radicular, o qual causa um decréscimo na turgescência e resulta na diminuição da expansão celular, reduzindo a taxa de crescimento das plantas (Bai et al., 2008; Khalid \& Silva, 2010). Essa redução do crescimento pode ainda ser explicada por limitações fotossintéticas, resultantes do fechamento estomático, e, concomitante, menor assimilação de $\mathrm{CO}_{2}$, (Debez et al., 2008; Taarit et al., 2010). Além disso, pode estar associada ao gasto energético envolvido na síntese de solutos orgânicos, necessários aos processos de compartimentalização e regulação do transporte de íns (Mendonça et al., 2007).

O aumento de sais na água de irrigação 
alterou o particionamento de matéria seca na planta, estabelecendo uma competição pela distribuição de fotoassimilados entre a parte aérea e as raízes da planta. Como consequência, verificou-se redução na partição de matéria seca para as raízes e aumento na proporção de partes aéreas, diminuindo, assim, a relação MSR/MSPA (Figura $2 \mathrm{E}$ e F). $P$. amboinicus e $P$. barbatus apresentaram redução linear da relação MSR/MSPA em função do aumento da CEa, sendo que as maiores quedas foram verificadas no ambiente a pleno sol. Para $P$. grandis a relação MSR/MSPA ajustou-se a um modelo quadrático, observando, nas plantas cultivadas em telado, maior alocação de matéria seca para as raízes na CEa de $1,6 \mathrm{dS} \mathrm{m}^{-1}$. Esta espécie, em pleno sol, obteve maior alocação de matéria seca para a parte aérea até a CEa de 3,1 dS m-1.

A tolerância de uma cultura à salinidade reflete a sua capacidade de suportar os efeitos do excesso de sais na zona radicular, sendo variável com fatores do solo e do clima (Qadir et al., 2008). Portanto, a tolerância à salinidade é descrita em termos relativos e, geralmente, dividida em quatro classes: tolerante, moderadamente tolerante, moderadamente sensível e sensível (Maas \& Hoffman, 1977; Fageria et al., 2010).

Na classificação para MSR, $P$. grandis e $P$. amboinicus, expostas a telado, apresentaram as menores perdas percentuais, sendo classificadas na CEa de 4,3 dS m-1 como moderadamente sensíveis, destacando que $P$. grandis apresentou-se como tolerante até CEa de 3,1 dS $\mathrm{m}^{-1}$ (Tabela 2). Já na CEa de $5,5 \mathrm{dS} \mathrm{m}^{-1}$ as três espécies se comportaram como sensíveis, registrando reduções superiores a $90 \%$ na MSR de $P$. amboinicus e $P$. barbatus expostas a pleno sol (Tabela 2).

$P$. grandis apresentou menor percentual de redução de matéria seca também na parte aérea das plantas, com classificação de tolerante até a CEa de 3,1 dS m-1 (Tabela 2). Nas CEa de 4,3 e 5,5

TABELA 2. Análise de tolerância à salinidade de espécies medicinais do gênero Plectranthus cultivadas em diferentes ambientes (telado e pleno sol), com base no percentual de redução da área foliar (AF), matéria seca da parte aérea (MSPA), matéria seca das raízes (MSR) e matéria seca total (MST). (Fortaleza-CE, UFC, 2012).

\begin{tabular}{|c|c|c|c|c|c|}
\hline \multirow{3}{*}{ Ambiente } & \multirow{3}{*}{ Espécies } & \multicolumn{4}{|c|}{ Redução em relação ao controle (CEa - 0,7 dS m-1) (\%) } \\
\hline & & $1,9 \mathrm{dS} \mathrm{m}^{-1}$ & $3,1 \mathrm{dS} \mathrm{m}^{-1}$ & $4,3 \mathrm{dS} \mathrm{m}^{-1}$ & $5,5 \mathrm{dS} \mathrm{m}^{-1}$ \\
\hline & & \multicolumn{4}{|c|}{ Matéria seca radicular (MSR) } \\
\hline & P. amboinicus & $19,84^{\top}$ & 39,68 мт & $59,53 \mathrm{~ms}$ & 79,37 s \\
\hline \multirow[t]{3}{*}{ Telado } & P. barbatus & $21,22^{\mathrm{Mт}}$ & $42,45^{\mathrm{ms}}$ & $63,67^{s}$ & $84,9 s$ \\
\hline & P. grandis & $2,12^{\top}$ & $17,52^{\top}$ & $46,21 \mathrm{~ms}$ & $88,17 \mathrm{~s}$ \\
\hline & P. amboinicus & $22,92^{\text {мт }}$ & $45,85^{\mathrm{ms}}$ & $68,78^{s}$ & $91,71^{s}$ \\
\hline \multirow[t]{4}{*}{ Pleno sol } & P. barbatus & $24,89^{м т}$ & $49,78 \mathrm{~ms}$ & 74,67 s & $99,56 s$ \\
\hline & P. grandis & $29,48^{\mathrm{MT}}$ & $51,54 \mathrm{~ms}$ & $66,16 s$ & $73,34 s$ \\
\hline & & \multicolumn{4}{|c|}{ Matéria seca parte aérea (MSPA) } \\
\hline & P. amboinicus & $14,51^{\top}$ & $29,02^{\text {мт }}$ & $43,54 \mathrm{~ms}$ & $58,05^{\mathrm{ms}}$ \\
\hline \multirow[t]{3}{*}{ Telado } & P. barbatus & $12,66^{\top}$ & $25,32^{\mathrm{Mт}}$ & $37,99^{\mathrm{мт}}$ & $50,65^{\mathrm{ms}}$ \\
\hline & P. grandis & $0^{\top}$ & $9,91^{\top}$ & $30,44 \mathrm{MT}$ & $60,99 s$ \\
\hline & P. amboinicus & $14,23^{\top}$ & $28,47 \mathrm{mт}$ & 42,71 мs & $56,95 \mathrm{~ms}$ \\
\hline \multirow[t]{4}{*}{ Pleno sol } & P. barbatus & $17,81^{\top}$ & $35,62^{\text {мт }}$ & $53,44 \mathrm{~ms}$ & $71,25^{s}$ \\
\hline & P. grandis & $1,64^{\top}$ & $12,09^{\top}$ & $31,36^{\mathrm{Mт}}$ & $59,45^{\mathrm{ms}}$ \\
\hline & & \multicolumn{4}{|c|}{ Matéria seca total (MST) } \\
\hline & P. amboinicus & $15,8^{\top}$ & $31,59 \mathrm{мт}$ & $47,39 \mathrm{~ms}$ & $63,18^{s}$ \\
\hline \multirow[t]{3}{*}{ Telado } & P. barbatus & $14,54^{\top}$ & 29,07 мт & $43,62^{\mathrm{ms}}$ & $58,14 \mathrm{~ms}$ \\
\hline & P. grandis & $0,47^{\top}$ & $12,05^{\top}$ & $34,766^{\mathrm{MT}}$ & $68,60^{s}$ \\
\hline & P. amboinicus & $16,51^{\top}$ & $33,01^{\mathrm{MT}}$ & $49,52^{\mathrm{ms}}$ & $66,03^{s}$ \\
\hline \multirow[t]{2}{*}{ Pleno sol } & P. barbatus & $18,04^{\top}$ & $36,09 \mathrm{Mт}$ & $54,13 \mathrm{~ms}$ & $72,17 \mathrm{~s}$ \\
\hline & P. grandis & $9,43^{\top}$ & $23,15^{\mathrm{MT}}$ & $41,16^{\mathrm{ms}}$ & $63,45^{s}$ \\
\hline
\end{tabular}

Rev. Bras. PI. Med., Campinas, v.16, n.4, p.839-849, 2014. 
dS $\mathrm{m}^{-1}$, foi observado maior redução da MSPA para $P$. barbatus cultivada em pleno sol, classificando-a nestes níveis de salinidade como moderadamente sensível e sensível à salinidade. A menor tolerância à salinidade da MSR em relação à MSPA (Tabela 2), possivelmente, pode estar associada à retenção de altas concentrações dos íons $\mathrm{Na}^{+}$e $\mathrm{Cl}$ nas raízes (Ashraf \& Orooj, 2006).

Considerando a MST, $P$. grandis cultivada em telado, apresentou até a CEa de 4,3 dS $\mathrm{m}^{-1}$ menor perda percentual $(34,76 \%)$, sendo moderadamente tolerante, enquanto que as demais espécies, independente do ambiente, foram moderadamente sensíveis à CEa de $4,3 \mathrm{dS} \mathrm{m}^{-1}$. A menor redução da MST obtida por $P$. grandis pode estar relacionada à capacidade dessa espécie em desenvolver mecanismos de tolerância à salinidade, proporcionando sua melhor aclimatação ao meio de cultivo salino. Ashraf \& Orooj (2006) atribuíram a tolerância moderada à salinidade de Trachyspermum ammi à manutenção da alta relação $\mathrm{K}+/ \mathrm{Na}^{+}$e $\mathrm{Ca}^{2+} / \mathrm{Na}^{+}$na parte aérea dessas plantas. A síntese de osmólitos compatíveis, alteração na via fotossintética, mudanças na estrutura das membranas e a indução de enzimas antioxidantes também são cruciais na tolerância das plantas à salinidade (Parida \& Das, 2005).

Os resultados apresentados neste trabalho mostram que o estresse salino induziu reduções significativas no crescimento das três espécies avaliadas, sendo que as maiores reduções ocorreram no crescimento das plantas expostas a pleno sol. O maior crescimento de plantas em ambientes com restrição de luz pode estar associado ao investimento em alongamento celular, visando o aproveitamento mais eficiente da energia luminosa, assim como também, pode ser beneficiado em decorrência do maior controle da temperatura foliar, favorecendo a menor taxa transpiratória, a maior abertura estomática, e a otimização da atividade fotossintética (Taiz \& Zeiger, 2013).

Considerando a produção de matéria seca total, verificou-se que a espécie $P$. grandis foi classificada como tolerante em ambiente telado, enquanto que as outras duas espécies se mostraram moderadamente tolerantes à salinidade até $3,1 \mathrm{dS}$ $\mathrm{m}^{-1}$ (Tabela 2). Porém, na salinidade de $5,5 \mathrm{dS} \mathrm{m}^{-1}$ todas se mostraram sensíveis ao excesso de sais na água de irrigação. Considerando o grau de redução na produção de matéria seca da parte área (parte de interesse comercial), poderia ser recomendado o cultivo da espécie $P$. grandis quando se dispõe de água de irrigação com CEa de até $3,1 \mathrm{dS} \mathrm{m}^{-1}$.

$\mathrm{Na}$ avaliação do IRC e dos íons inorgânicos, $\mathrm{Na}^{+}$e $\mathrm{K}^{+}$, verificaram-se efeitos significativos dos fatores isolados: ambiente, salinidade e espécies. Quanto à interação desses fatores não houve efeito significativo do ambiente vs salinidade para o IRC e para o íon $\mathrm{Na}^{+}$e do ambiente vs salinidade vs espécie para o IRC (Tabela 3 ).

O maior IRC no ambiente telado foi registrado para $P$. barbatus contrastando com $P$. grandis e $P$. amboinicus que não diferiram entre si pelo teste Tukey $(p<0,05)$ (Tabela 4$)$. Já a pleno sol, as maiores médias de IRC foram registradas para $P$. amboinicus e $P$. barbatus que não diferiram entre si pelo teste de Tukey $(p<0,05)$ (Tabela 4). A intensidade luminosa não interferiu no IRC das plantas de P. grandis (Tabela 4). Resultados semelhantes foram apresentados por Castro et al. (2005) em plantas de Mikania glomerata, e por Pinto et al. (2007) em plantas de Aloysia gratissima.

Houve redução no IRC de todas as espécies com o aumento da CEa (Figura 3). Essa redução foi da ordem de $44,56 \%$ para P. amboinicus, $24,95 \%$ para $P$. grandis e $23,88 \%$ para $P$. barbatus entre os níveis de CEa de 0,7 e 5,5 dS m-1. Redução nos teores de clorofila em plantas crescidas em meio salino tem

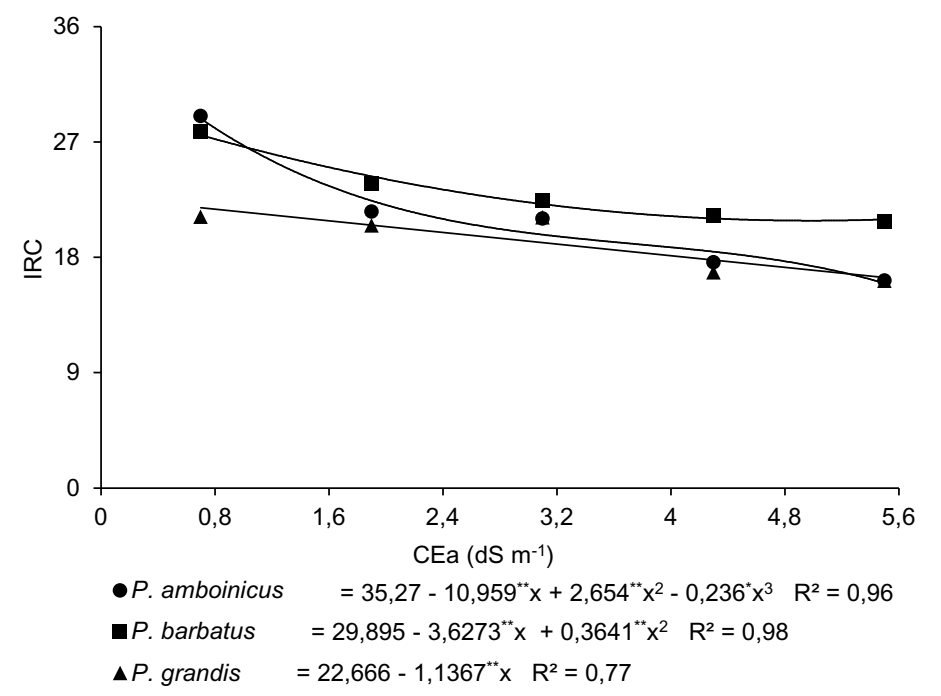

FIGURA 3. Índice relativo de clorofila (IRC) em três espécies medicinais do gênero Plectranthus em função de níveis crescentes de condutividade elétrica na água de irrigação. (Fortaleza-CE, UFC, 2012).

Rev. Bras. PI. Med., Campinas, v.16, n.4, p.839-849, 2014. 
TABELA 3. Resumo da análise de variância para índice relativo de clorofila (IRC), teor de sódio ( $\left.\mathrm{Na}^{+}\right)$e potássio $\left(\mathrm{K}^{+}\right)$nas folhas de três espécies medicinais do gênero Plectranthus cultivadas em telado com $50 \%$ de luminosidade e pleno sol, e submetidas a níveis crescentes de condutividade elétrica na água de irrigação. (Fortaleza-CE, UFC, 2012).

\begin{tabular}{|c|c|c|c|c|}
\hline \multirow{2}{*}{ FV } & \multirow{2}{*}{ GL } & \multicolumn{3}{|c|}{ Quadrados médios } \\
\hline & & IRC & $\mathrm{Na}^{+}$ & $\mathrm{K}^{+}$ \\
\hline$A$ & 1 & $33,127^{* *}$ & $5,50^{* *}$ & $317,90^{* *}$ \\
\hline $\operatorname{Res}(a)$ & 6 & 1,883 & 0,28 & 21,55 \\
\hline B & 4 & $256,106^{* *}$ & $196,34^{* *}$ & $1239,44^{* *}$ \\
\hline$A \times B$ & 4 & $6,912^{\mathrm{Ns}}$ & $0,49^{\mathrm{Ns}}$ & $474,57^{* *}$ \\
\hline $\operatorname{Res}(b)$ & 24 & 2,615 & 0,53 & 25,97 \\
\hline C & 2 & 164,526 & $45,69^{* *}$ & $1273,41^{* *}$ \\
\hline$A \times C$ & 2 & $58,905^{* *}$ & $40,11^{*}$ & $1620,60^{* *}$ \\
\hline$B \times C$ & 8 & $26,978^{* *}$ & $8,28^{* *}$ & $159,06 *$ \\
\hline$A \times B \times C$ & 8 & $2,128^{\mathrm{NS}}$ & $4,39^{* *}$ & $301,35 *$ \\
\hline $\operatorname{Res}(c)$ & 60 & 3,618 & 1,01 & 34,27 \\
\hline CV(a) (\%) & - & 6,49 & 8,62 & 11,03 \\
\hline CV(b) $(\%)$ & - & 7,65 & 11,77 & 12,11 \\
\hline $\mathrm{CV}(\mathrm{c})(\%)$ & - & 9,0 & 16,28 & 13,91 \\
\hline
\end{tabular}

FV - fontes de variação; GL - graus de liberdade; A - ambiente; B - salinidade; C - espécies; CV - coeficiente de variação; **, ${ }^{*}, \mathrm{NS}$ significativo pelo teste $\mathrm{F}$ a $1 \%, 5 \%$ e não significativo, respectivamente.

TABELA 4. Índice relativo de clorofila (IRC) aos 50 DAT em folhas de três espécies medicinais do gênero Plectranthus, sob diferente luminosidade. (Fortaleza-CE, UFC, 2012).

\begin{tabular}{ccc}
\hline \multirow{2}{*}{ Espécie } & \multicolumn{2}{c}{ IRC (Unidade - SPAD) } \\
\cline { 2 - 3 } & Telado & Pleno sol \\
\hline P. grandis & $19,56 \mathrm{aB}$ & $18,72 \mathrm{aB}$ \\
P. barbatus & $24,99 \mathrm{aA}$ & $21,41 \mathrm{bA}$ \\
P. amboinicus & $20,45 \mathrm{bB}$ & $21,72 \mathrm{aA}$ \\
\hline
\end{tabular}

Médias seguidas da mesma letra, minúsculas linhas e maiúsculas colunas, não diferem pelo teste de Tukey $(p<0,05)$.

sido atribuída ao aumento da atividade da clorofilase, induzindo a destruição da estrutura do cloroplasto e à instabilidade de complexos de proteínas do pigmento (Santos, 2004; Jamil et al., 2007).

$\mathrm{O}$ teor de $\mathrm{Na}^{+}$nas folhas, para todas as espécies, aumentou em função do acréscimo da CEa nos dois ambientes de estudo (Figura 4 ). No ambiente com telado, a espécie $P$. amboinicus apresentou o maior teor de $\mathrm{Na}^{+}$seguida por $P$. barbatus e $P$. grandis, enquanto que a pleno sol o maior teor deste íon foi em $P$. barbatus, seguido por $P$. amboinicus e P. grandis (Figura 4). Em contrapartida, verificou-se redução nos teores de $\mathrm{K}^{+}$, especialmente quando se compara a menor CEa $\left(0,7 \mathrm{dS} \mathrm{m}^{-1}\right)$ e a maior CEa (5,5 dS $\left.\mathrm{m}^{-1}\right)$ (Figura 4).

Distúrbios nutricionais sob salinidade reduzem o crescimento das plantas afetando a disponibilidade, o transporte e a partição de nutrientes. A redução na absorção de $\mathrm{K}^{+}$na presença elevada de $\mathrm{Na}^{+}$deve-se a competição por sítios no transporte de proteínas e, através de processos intracelulares, ainda, não são bem compreendidos. Como o $\mathrm{Na}^{+}$não substitui as funções específicas do $\mathrm{K}^{+}$, tais como a manutenção da atividade enzimática, a neutralização de ânions no citosol e cloroplastos, e a manutenção do $\mathrm{pH}$ em níveis adequados para o funcionamento da célula, é provável que as alterações no crescimento e na capacidade fotossintética das plantas em função do aumento da salinidade, estejam relacionadas à reduzida absorção do $\mathrm{K}^{+} \mathrm{e}$, concomitante redução da concentração no citoplasma e cloroplastos (Tavakkoli et al., 2011; Taiz \& Zeiger, 2013).

A indução do estresse salino através do uso de água com sais de $\mathrm{NaCl}, \mathrm{CaCl}_{2} \cdot 2 \mathrm{H}_{2} \mathrm{O}$ e $\mathrm{MgCl}_{2} \cdot 6 \mathrm{H}_{2} \mathrm{O}$ afetou a partição de matéria seca nas

Rev. Bras. Pl. Med., Campinas, v.16, n.4, p.839-849, 2014. 

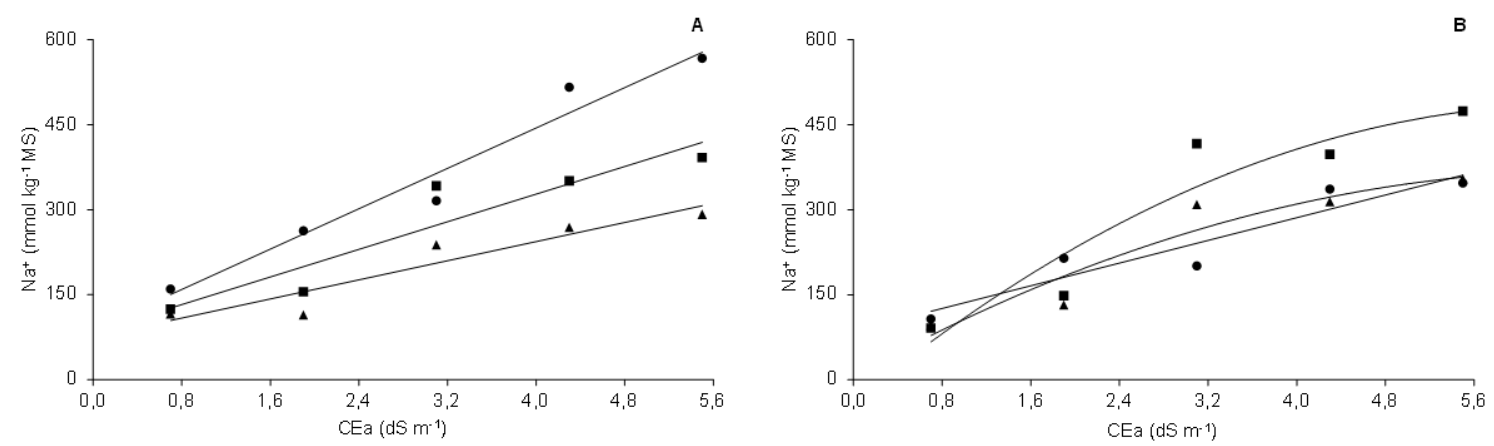

-P. amboinicus $\hat{y}=88,335+89,027^{\prime \prime} \times \quad R^{2}=0,90$

- $P$. barbatus $\hat{y}=83,995+60,893^{\prime \prime} \times R^{2}=0,87$

AP. grandis $\hat{\mathrm{y}}=75,139+42,141^{* *} \times R^{2}=0,88$

-P. amboinicus $\hat{\mathrm{y}}=85,828+50,123^{\mathrm{x} \times} \times \mathrm{R}^{2}=0,89$

- P. barbatus $\hat{\mathrm{y}}=-39,524+160,94^{\mathrm{4x}} \mathrm{x}-12,319^{\mathrm{xx}} \mathrm{x}^{2} \quad \mathrm{R}^{2}=0,89$

AP. grandis $\hat{y}=6,6042+108,15^{n x} x-8,059^{x} x^{2} \quad R^{2}=0,91$
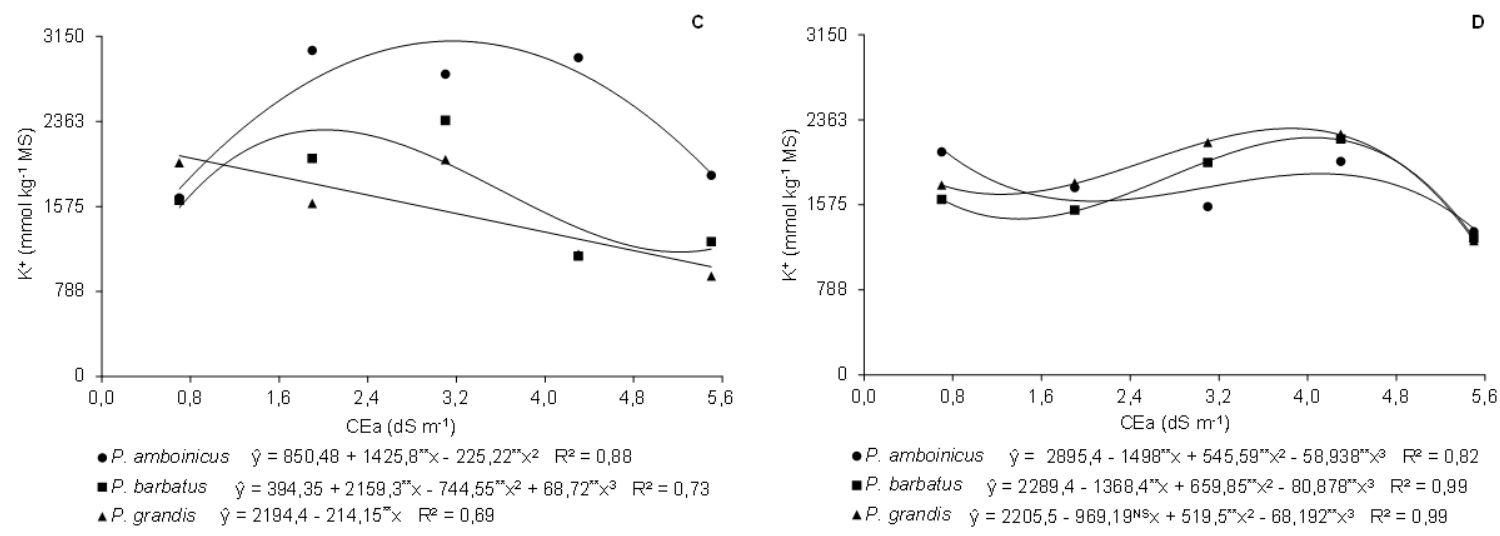

FIGURA 4. Teor de $\mathrm{Na}^{+}$e $\mathrm{K}^{+}$em folhas de três espécies medicinais do gênero Plectranthus cultivadas em telado com $50 \%$ de luminosidade ( $A$ e $C$ ) e em pleno sol (B e D) e submetidas a níveis crescentes de condutividade elétrica na água de irrigação. (Fortaleza-CE, UFC, 2012).

plantas, sendo as raízes mais afetadas que a parte aérea. Considerando-se a produção de matéria seca total, verifica-se que as três espécies estudadas se mostraram tolerantes ou moderadamente tolerantes à salinidade de até $3,1 \mathrm{dS} \mathrm{m}^{-1}$, porém na salinidade de $5,5 \mathrm{dS} \mathrm{m}^{-1}$ todas se mostraram sensíveis ao excesso de sais na água de irrigação. Com o aumento da CEa houve um aumento expressivo no teor de $\mathrm{Na}^{+}$, enquanto o teor de $\mathrm{K}^{+} \mathrm{e}$ o IRC foram reduzidos.

\section{AGRADECIMENTO}

Os autores agradecem à Fundação Cearense de Apoio ao Desenvolvimento Científico e Tecnológico (FUNCAP) pela concessão da bolsa de estudo e ao Instituto Nacional de Ciência e Tecnologia em Salinidade (INCTSal/CNPq) pelo suporte financeiro.

\section{REFERÊNCIA}

ABDEL-MOGIB, M.; ALBAR, H.A.; BAtTERJEe, S.M. Review: Chemistry of the Genus Plectranthus.
Molecules, v.7, n.2, p.271-301, 2002.

ALASBAHI, R.H.; MELZIG, M.F. Plectranthus barbatus: A Review of Phytochemistry, Ethnobotanical Uses and Pharmacology - Part 1. Planta Medica, v.76, n.7, p.653-661, 2010.

ASHRAF, M.; OROOJ, A. Salt stress effects on growth, ion accumulation and seed oil concentration in an arid zone traditional medicinal plant ajwain (Trachyspermum ammi [L.] Sprague). Journal of Arid Environments, v. 64, n. 2, p.209-220, 2006.

AYERS, R.S.; WESTCOT, D.W. A qualidade da água na agricultura. Trad. GHEYI, H.R. et al., Campina grande: UFPB, 1999. 153p.

BANDEIRA, J.M. et al. Composição do óleo essencial de quatro espécies do gênero Plectranthus. Revista Brasileira de Plantas Medicinais, v.13, n.2, p.157164, 2011.

BAI, W. et al. Some Physiological Responses of Chinese Iris to Salt Stress. Pedosphere, v.18, n.4, p.454-463, 2008.

BENINCASA, M.M.P. Análise de crescimento de plantas (noções básicas). 2ed Jaboticabal: FUNEP, 41p. 2003.

BERNSTEIN, N.; KRAVCHIK, M.; DUDAI, N. Salinityinduced changes in essential oil, pigments and salts accumulation in sweet basil (Ocimum basilicum) in relation to alterations of morphological development. 
Annals of Applied Biology, v.156, n.2, p.167-177, 2010.

CASTRO, E.M. et al. Aspectos anatômicos e fisiológicos de plantas de guaco submetidas a diferentes fotoperíodos. Horticultura Brasileira, Brasília, v.23, n.3, p.846-850, 2005.

CATALDO, D.A. et al. Rapid colorimetric determination of nitrate in plant tissue by nitration of salicylic acid. Communications in Soil Science and Plant Analysis, v.6, p.71-80, 1975.

DEBEZ, A. et al. Relationship between the photosynthetic activity and the performance of Cakile maritime after long-term salt treatment. Physiologia Plantarum, v.133, n.2, p.373-385, 2008.

EMPRESA BRASILEIRA DE PESQUISA AGROPECUÁRIA - EMBRAPA. Manual de métodos de análises de solo. Rio de Janeiro, Ministério da Agricultura e do Abastecimento, 2.ed, 1997, 212p.

FAGERIA, N.K.; SOARES FILHO, W.S; GHEYI, H. $\mathrm{R}$. Melhoramento genético vegetal e seleção de cultivares tolerantes à salinidade. In: GHEYI, H. R.; DIAS, N. S.; LACERDA, C. F. (eds). Manejo da salinidade na agricultura: estudos básicos e aplicados. Fortaleza, INCTSal, 2010. p.205-216.

FERREIRA, D.F. Sisvar versão 5.3. Sistemas de análises de variância para dados balanceados: programa de análises estatísticas e planejamento de experimentos. Lavras, MG, Universidade Federal de Lavras, 2010.

KHALID, A.; SILVA, J.A.T. Yield, essential oil and pigment content of Calendula officinalis L. flower heads cultivated under salt stress conditions. Scientia Horticulturae, v.126, n.2, p.297-305, 2010. KOYRO, H.W. Effect of salinity on growth, photosynthesis, water relations and solute composition of the potential cash crop halophyte Plantago coronopus (L.). Environmental and Experimental Botany, v.56, n.2, p.136-146, 2006.

JAMIL, M. et al. Salinity reduced growth PS2 photochemistry and chlorophyll content in radish. Scientia Agricola. v. 64, n.2, p.111-118, 2007.

LUKHOBA, C.W.; SIMMONDS, M.S.J.; PATON, A.J. Plectranthus: A review of ethnobotanical uses. Journal of Ethnopharmacology, v.103, n.1, p.1-24, 2006.

MAAS, E.V.; HOFFMAN, G.J. Crop salt tolerance current assessment. Journal of the Irrigation and Drainage Division, ASCE 103, p.115-134, 1977.

MALAVOLTA, E.; VITTI, G.C.; OLIVEIRA, S.A. Avaliação do estado nutricional das plantas: Princípios e Aplicações. Associação Brasileira para Pesquisa da Potassa e do Fosfato. Piracicaba, SP, 1989. 201p.

MENDONÇA, A.V.R. et al. Características biométricas de mudas de eucalyptus sp sob estresse salino. Revista
Árvore, v.31, n.3, p.365-372, 2007.

MUNNS, R. Genes and salt tolerance: bringing them together. New Phytologist, v.167, n.3 p.645-663, 2005.

MUNNS, R.; TESTER, M. Mechanisms of Salinity Tolerance. Annual Reviews of Plant Biology, v.59, p.651-681, 2008.

PARIDA, A.K.; DAS, A.B. Salt tolerance and salinity effects on plants: a review. Ecotoxicology and Environmental Safety, v.60, n.3, p.324-349, 2005.

PINTO J.E.B.P. et al. Aspectos morfofisiológicos e conteúdo de óleo essencial de plantas de alfazemado-Brasil em função de níveis de sombreamento. Horticultura Brasileira, v.25, n.2, p.210-214, 2007.

QADIR, M. et al. Productivity enhancement of saltaffected environments through crop diversification. Land Degradation \& Development, v.19, n.4, p.429-453, 2008.

RHOADES, J.P.; KANDIAH, A.; MASHALI, A.M. The use saline waters for crop production. Trad.: GHEYI, $H$. R.; SOUSA, J. R.; QUEIROZ, J. E. Campina Grande: UFPB, 2000. 117p.

RICE, L. J. et al. Plectranthus: A plant for the future? South African Journal of Botany, v.77, n.4, p.947959, 2011.

SABRA, A.; DAAYF, F.; RENAULT, S. Differential physiological and biochemical responses of three Echinacea species to salinity stress. Scientia Horticulturae, v.135, n.9, p.23-31, 2012.

SAIRAM, R.K.; TYAGY, A. Physiology and molecular biology of salinity stress tolerance in plants. Current Science, v.86, n.3, p.407-421, 2004.

SANTOS, C.V. Regulation of chlorophyll biosynthesis and degradation by salt stress in sunflower leaves. Scientia Horticulturae, v.103, n.1, p.93-99, 2004.

SILVEIRA, J.A.G. et. al. Salt resistance in two cashew species is associated with accumulation of organic and inorganic solutes. Acta Physiologiae Plantarum, v.34, n.5, p.1629-1637, 2012.

TAARIT, M.B. et al. Changes in fatty acid and essential oil composition of sage (Salvia officinalis L.) leaves under $\mathrm{NaCl}$ stress. Food Chemistry, v.119, n.3, p.951-956, 2010.

TAIZ, L.; ZEIGER, E. Fisiologia Vegetal, Porto Alegre, ARTMED. $5^{\circ}$ ed., 2013, 918p.

TAVAKKOLI, E. et al. Additive effects of $\mathrm{Na}+$ and $\mathrm{Cl}-$ ions on barley growth under salinity stress. Journal of Experimental Botany, v.62, n.6, p.2189-2203, 2011.

TOUNEKTI, T. et al. Canopy position determines the photoprotective demand and antioxidant protection of leaves in salt-stressed Salvia officinalis L. plants. Environmental and Experimental Botany, v.78, p.146-156, 2012. 